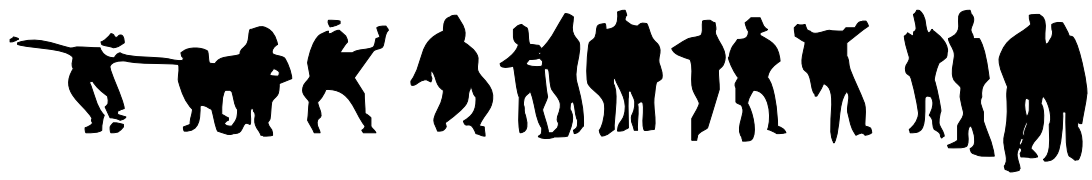

\section{Cadre d'analyse de la personnalisation de l'apprentissage dans les cours en ligne ouverts et massifs (CLOM)}

\author{
- Rim BEJAOUI, Gilbert PAQUETTE, Josianne BASQUE et France \\ HENRI (Laboratoire LICÉ, Centre de recherche LICEF, Télé- \\ université du Québec)
}

ש RÉSUMÉ • Nous présentons un cadre d'analyse de la personnalisation dans les CLOM. Fondé théoriquement, ce cadre comprend une ontologie des diverses propriétés de personnalisation de l'apprentissage qui peuvent s'appliquer dans les CLOM, ainsi qu'une grille d'analyse de ces propriétés. La grille devient pour le concepteur de CLOM un instrument dans sa prise de décision quant aux options de personnalisation de l'apprentissage envisageables. Le cadre a fait l'objet d'une validation par des experts, dont nous présentons les résultats.

- MOTS-CLÉS •CLOM, apprentissage personnalisé, ontologie, grille d'analyse, adaptabilité du scénario, assistance à l'apprenant, aide à l'autogestion de l'apprentissage.

- ABSTRACT - We present a theoretically founded framework for analyzing personalization in MOOCs which includes an ontology of the various properties of learning personalization that can be applied in MOOCs and an analytical grid of these properties. The grid can assist the MOOC designer in his decision-making for learning personalization. We also present the validation results of the framework by experts.

KEYWORDS - MOOC, personalized learning, ontology, analytical grid, scenario adaptability, learner support, learning self-regulation. 


\section{Introduction}

L'expansion du phénomène des cours en ligne ouverts et massifs (CLOM) depuis le début des années 2010 a donné lieu à un nombre croissant de travaux de recherche. Les chercheurs déplorent notamment le manque de soutien fourni aux concepteurs de CLOM (Alario-Hoyos et al., 2014), alors que ce type de formation en ligne pose des défis nouveaux aux plans pédagogique, logistique, technologique et administratif. Certains travaillent à développer des instruments qui permettraient d'analyser dans quelle mesure les CLOM respectent des principes associés à un design pédagogique de qualité (Raposo-Rivas, et al., 2015), (Yousef et al., 2014), mais ces travaux demeurent exploratoires et leurs fondements théoriques peu explicités. En particulier, la question de la personnalisation de l'apprentissage offerte dans les CLOM demeure encore peu abordée dans ces travaux. Celle-ci se pose pourtant avec acuité compte tenu du caractère massif et de la grande hétérogénéité des publics cibles en termes de compétences et de motivations, ce qui peut faire obstacle à un tutorat personnalisé.

Plusieurs auteurs soulèvent ainsi le besoin urgent de personnaliser davantage l'apprentissage dans les CLOM (Kravcik et al. 2014), notamment pour y augmenter la rétention des participants (Blanco et al., 2013), (Miranda et al., 2013). Un CLOM mal adapté aux connaissances, aux compétences, aux préférences et aux objectifs de l'apprenant remplira mal ses fonctions d'apprentissage et risque d'être abandonné en cours de route. Des études menées à ce jour définissent sommairement un ensemble de pratiques pédagogiques visant à améliorer la qualité pédagogique des CLOM, notamment en y soutenant un apprentissage personnalisé, mais sans approfondir cette dernière dimension. D'autres études décrivent des démarches plus ou moins opérationnelles pour la conception de CLOM adaptatifs (Blanco et al., 2013), (Santos et al., 2014) et d'autres encore proposent des solutions de personnalisation mais qui n'ont pas encore été expérimentées dans des plateformes de CLOM (Clerc, 2014), (Halimi et al., 2014), (Henning et al., 2014), (Mott, 2010), (Paquette et al., 2015), (Pedro et al., 2012), (Shaw, 2013), (Szafir et Mutlu, 2013), (Yang et al., 2014). Il y a donc un besoin d'énoncer les fondements théoriques de la personnalisation, d'en cerner le concept et les manifestations de propriétés et de développer des outils à l'usage du concepteur de CLOM afin de l'assister dans sa prise de décision quant aux options de personnalisation de l'apprentissage qu'il 
peut envisager. C'est à ce besoin que notre projet de recherche (Bejaoui, 2017) vise à répondre.

Dans nos travaux présentés ici, en nous appuyant sur les écrits théoriques liés au domaine, nous avons identifié deux groupes de propriétés des CLOM soutenant un apprentissage personnalisé. Une ontologie, élaborée à partir de ces groupes de propriétés, a servi de modèle opérationnel pour l'élaboration d'une grille permettant d'évaluer le degré de personnalisation de l'apprentissage dans un CLOM. Cette grille a, par la suite, servi de base au développement d'un prototype d'assistance à la conception de CLOM personnalisables (CLOMp). Ces derniers travaux (grille et prototype) ont fait l'objet d'une validation par des experts en personnalisation de l'apprentissage et en ingénierie pédagogique. Dans cet article, nous nous limitons à présenter l'ontologie et la grille d'analyse, ainsi que les résultats de la validation de cette dernière par les experts.

Dans la première section de cet article, nous exposons les éléments théoriques qui ont orienté le travail de conception de la grille d'analyse. Dans les sections qui suivent, nous présentons notre ontologie d'un scénario pédagogique de CLOMp, notre grille d'analyse, les résultats de l'application de notre grille sur un exemple de CLOM et finalement les résultats de la validation de la grille.

\section{Cadre théorique}

La notion de personnalisation de l'apprentissage englobe celle de l'individualisation de l'enseignement, où l'étudiant peut progresser à son rythme, et celle de la différentiation de l'apprentissage, où il peut choisir entre diverses méthodes d'apprentissage selon ses préférences ou ses caractéristiques propres. La personnalisation va plus loin dans un environnement où, aussi bien, les objectifs d'apprentissage et le contenu, que la méthode pédagogique et le rythme varient selon les choix de l'apprenant (USDE, 2010). Les adaptations effectuées dans l'environnement d'apprentissage pour convenir aux besoins et préférences des apprenants peuvent aussi bien être effectuées par un agent externe à l'apprenant que par l'apprenant lui-même, et, dans les deux cas, les technologies peuvent faciliter grandement le travail d'adaptation. Ainsi, nous distinguons trois approches de personnalisation de l'apprentissage dans un environnement d'apprentissage. La première est celle de la personnalisation opérée par un agent externe à l'apprenant (système tutoriel ou conseiller, ou acteur humain) utilisant, par exemple, 
les systèmes adaptatifs, les systèmes tutoriels intelligents ou les systèmes de recommandation. La deuxième est celle de la personnalisation opérée par l'apprenant utilisant, par exemple, le concept d'environnement personnel d'apprentissage (EPA). La troisième approche se caractérise par des propositions de personnalisation hybrides, combinant les deux premières approches.

Pour élaborer notre cadre d'analyse de la personnalisation de l'apprentissage, nous nous sommes intéressés au concept de pédagogie ouverte (C. Paquette, 1992), c'est-à-dire une pédagogie qui laisse à l'apprenant un certain degré de liberté dans sa démarche d'apprentissage lui permettant d'en personnaliser lui-même plusieurs aspects. Cette centration sur $l^{\prime}$ " ouverture pédagogique» implique une mise à disposition dans l'environnement d'apprentissage de ressources destinées à l'apprenant afin de le soutenir dans l'autogestion de sa démarche d'apprentissage (Bandura, 1997) et la création de son environnement personnel d'apprentissage (EPA) (Henri, 2014). Les théories de l'inscription sociale de l'apprentissage (Bandura, 1977), (Hutchins, 1995, chap. 9), (Pea, 1993), (Siemens, 2004), (Vygotski, 1978) ajoutent à l'idée d'ouverture pédagogique celle de l'apport des interactions sociales dans l'apprentissage, vues comme étant ancrées dans un contexte socioculturel donné et dans un contexte d'apprentissage signifiant pour l'apprenant. Cette idée se trouve au cœur des Anchored Open Courses (Baker et Surry, 2013) dont les principes pédagogiques ont inspiré en partie notre travail. Notre cadre théorique s'appuie également sur les modèles et approches développés dans le domaine de l'ingénierie pédagogique (IP), notamment la méthode d'ingénierie des systèmes d'apprentissage MISA (Paquette, 2002a) pour son approche centrée sur la modélisation et pour son modèle de scénario pédagogique, ainsi que l'approche théorique du meta-design (Fischer, 2007) et le modèle Seeding-Evolutionary Growth-Reseeding (Fischer, 2012), (Fischer et Ostwald, 2002) pour leur idée d'apprenantconcepteur. Pour finir, nous nous sommes intéressés aux ontologies en tant qu'outils de modélisation formelle pour l'analyse, la conception, le développement et la personnalisation des environnements numériques d'apprentissage (ENA).

\section{Ontologie d'un scénario pédagogique de CLOM soutenant un apprentissage personnalisé}

Pour qu'un CLOM soit réellement ouvert, le concepteur doit élaborer un scénario pédagogique pouvant être personnalisé par un ou plusieurs 
acteurs du CLOM, à savoir un facilitateur, un agent logiciel, un apprenant ou un groupe d'apprenants. Diverses fonctionnalités intégrées dans les plateformes de CLOM peuvent soutenir ces approches de personnalisation. Elles ne sont cependant pas forcément exploitées par les concepteurs de CLOM, qui ne disposent pas d'une vue d'ensemble des possibilités qui s'offrent à eux en matière de personnalisation de l'apprentissage dans les CLOM. Dans un effort pour fournir une telle vue d'ensemble, nous avons défini deux groupes de propriétés technopédagogiques soutenant la personnalisation de l'apprentissage dans un CLOM et construit une ontologie d'un scénario pédagogique de CLOMp intégrant ces deux groupes de propriétés.

\subsection{Les deux groupes de propriétés soutenant la personnalisation de l'apprentissage}

Les propriétés d'adaptation des composantes du scénario pédagogique du CLOM font référence au degré d'adaptabilité (ou de modificabilité) de certaines composantes du scénario pour convenir aux besoins spécifiques des apprenants. Ces adaptations peuvent être accomplies par l'un des quatre principaux acteurs du CLOM (facilitateur, agent logiciel, apprenant ou groupe d'apprenants) et portent, par exemple, sur les compétences visées dans le CLOM, le moment de démarrage d'une activité d'apprentissage, le mode de collaboration entre les apprenants.

Les propriétés d'assistance et d'aide à l'autogestion de l'apprentissage font référence au degré avec lequel le CLOM offre des ressources d'Assistance et d'Aide à l'Autogestion de l'Apprentissage (que nous nommons ressources4A), aidant les apprenants tant à autogérer leur processus de développement des compétences disciplinaires visées dans le CLOM qu'à améliorer leur autonomie dans leur expérience éducative. Ceci inclut la mise à disposition de l'apprenant, par un agent logiciel ou un acteur humain, de ressources variées telles qu'un outil d'autodiagnostic de ses compétences, un outil de planification du travail, un e-portfolio, etc.

\subsection{Description de I'ontologie}

Notre ontologie d'un scénario pédagogique de CLOMp a été réalisée sur plusieurs itérations, à l'aide du multi-éditeur G-MOT (Paquette, 2002b). Dans une première itération, notre modèle ontologique comportait uniquement les deux groupes de propriétés présentés à la section 2.1. Par la suite, nous avons fait évoluer ce modèle vers une ontologie reliant le modèle de scénario pédagogique de la méthode d'ingénierie des systèmes 
Rim BEJAOUI, Gilbert PAQUETTE, Josianne BASQUE, France HENRI

d'apprentissage MISA aux groupes de propriétés soutenant la personnalisation de l'apprentissage. Nous avons choisi l'outil d'édition GMOT pour plusieurs raisons. D'abord, il permet l'édition d'ontologies à l'aide d'un formalisme simple et offre des capacités d'exportation et d'importation d'ontologies répondant à la norme OWL-DL. De plus, il est intégré dans la plateforme TELOS (Paquette et al., 2007), sur laquelle nous avons développé notre prototype. Dans ce qui suit, par souci de concision, nous montrons uniquement le niveau supérieur du modèle de l'ontologie (figure 1) et décrivons les sous-niveaux (c'est-à-dire les sous-modèles) des classes les plus importantes. La version complète du modèle de l'ontologie inclut vingt-six sous-modèles (voir à https://drive.google.com/file/d/0Bw9IV9UOtZd7LUgzQVFsbnQ3X1U/vie $\mathrm{w}$ ?usp=sharing). Les classes de l'ontologie sont indiquées en italique dans le texte qui suit.

Comme le montre la figure $1^{1}$, au centre du niveau supérieur de l'ontologie se trouve la classe principale Scénario pédagogique de CLOMp. Un scénario est composé d'activités d'apprentissage, de ressources pédagogiques et d'acteurs, et est caractérisé par un ensemble de propriétés (par ex. liste des compétences, ordonnancement des activités d'apprentissage). On y voit également qu'une activité d'apprentissage est régie par l'acteur réalisateur de l'activité d'apprentissage et est caractérisée par un ensemble de propriétés (par ex. moment de démarrage, durée, modalité de reprise). 


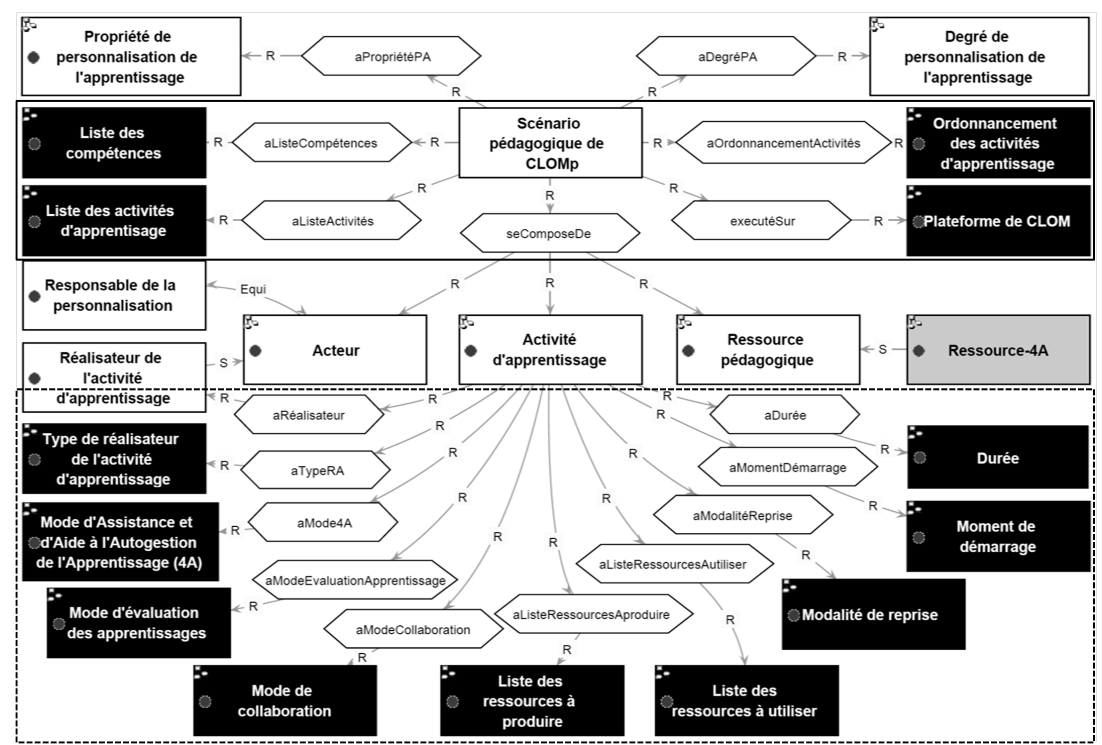

Figure $1 \cdot$ Niveau supérieur du modèle de l'ontologie d'un scénario pédagogique de CLOMp

\subsubsection{Composantes adaptables et ressources-4A du scénario pédagogique de CLOMp}

Dans notre ontologie, les classes représentées par les rectangles noirs (voir figure 1) constituent l'ensemble des composantes adaptables du scénario pédagogique de CLOMp. Certaines composantes concernent le scénario global dit « de haut niveau " (voir encadré en trait continu en haut de la figure 1) et d'autres concernent les activités incluses dans le scénario (voir encadré en trait discontinu en bas de la figure 1). Ces dernières classes sont instanciables à des valeurs qui varient selon les spécificités du scénario pédagogique de CLOMp et de ses activités d'apprentissage. Les Ressource-4A sont destinées aux apprenants dans le but de leur porter Assistance et/ou de les Aider à Autogérer leur Apprentissage. Représentées à l'aide du rectangle gris dans la figure 1, elles sont un type particulier de ressources pédagogiques (voir lien de spécialisation « $\mathrm{S}$ »). Le sous-modèle de la classe Ressource-4A (non présenté dans la figure 1) définit quatre types de ressources, comme indiqué dans notre glossaire (voir à https://drive.google.com/file/d/OBw9IV9UOtZd7Q1MzTTI3YldKVVE/vie w? usp=sharing) : 
- la classe Guide-4A regroupe les ressources pédagogiques comportant des descriptions et des consignes relatives aux activités d'apprentissage, aux instruments, aux outils et aux services du CLOMp et dont la fonction est d'assister l'apprenant et/ou de l'aider à autogérer son apprentissage (par ex. guide d'animation du forum du CLOMp) ;

- la classe Instrument-4A regroupe les ressources pédagogiques produites/utilisées par l'un des acteurs du CLOMp et présentant à l'apprenant des informations pour améliorer ses connaissances relatives à l'autogestion de son apprentissage (par ex. matériel pédagogique présentant les diverses habiletés d'autogestion);

- la classe Outil-4A regroupe les ressources pédagogiques permettant à l'apprenant de percevoir ou de transformer l'information du CLOMp pour l'aider à autogérer son apprentissage (par ex. e-portfolio, graphique de progression);

- la classe Service-4A regroupe les ressources pédagogiques prenant la forme de messages fournis par un agent humain ou informatique visant à assister l'apprenant lorsqu'il rencontre des problèmes et/ou à l'aider à autogérer son apprentissage (par ex. messages personnalisés envoyés à l'apprenant par un formateur ou générés par le système).

\subsubsection{Les propriétés et le degré de personnalisation de l'apprentissage du scénario pédagogique de CLOMp}

Dans notre ontologie (voir figure 1), la classe Propriété de personnalisation de l'apprentissage englobe l'ensemble des propriétés définies dans la section 2.1 et permet d'associer un certain degré de personnalisation de l'apprentissage au scénario pédagogique du CLOM. Ce degré est représenté, dans l'ontologie, par la classe Degré de personnalisation de l'apprentissage et peut correspondre à un degré de personnalisation " très présent ", « assez présent », " moyennement présent », "peu présent " ou «absent». Il peut être déduit et raffiné en plusieurs sous-indicateurs grâce à la présence ou à l'absence des instances des classes Composante adaptable et Ressource- $4 A$ et des valeurs de pondération qui leur sont associées, que nous présentons dans la section qui suit.

\section{Grille d'analyse du degré de personnalisation de l'apprentissage dans un CLOM}

Cette section présente notre grille d'analyse du degré de personnalisation de l'apprentissage dans un CLOM. Cette dernière se compose : 
- (1) des groupes de propriétés technopédagogiques qui soutiennent, à des degrés plus ou moins élevés, la personnalisation de l'apprentissage dans un CLOM,

- (2) de règles de pondération associées à ces propriétés,

- (3) d'une méthode de calcul des scores de personnalisation de l'apprentissage (PA) prenant en compte la présence/absence de ces propriétés dans un CLOM et les valeurs de pondération associées à ces dernières,

- (4) de règles d'exclusion mutuelle des propriétés d'adaptation des composantes du CLOM,

- (5) des valeurs maximales des scores de PA d'un CLOM, et

- (6) d'une méthode d'interprétation des scores de PA obtenus lorsqu'un CLOM est analysé à l'aide de notre grille.

\subsection{Propriétés techno-pédagogiques soutenant la personnalisation de l'apprentissage dans un CLOM}

Notre grille comprend au total 121 propriétés soutenant la personnalisation de l'apprentissage dans le CLOM, dont 21 propriétés d'adaptation de composantes de haut niveau du CLOM, 73 propriétés d'adaptation de composantes liées aux activités d'apprentissage et 27 exemples de ressources- $4 A$.

Pour définir le degré de personnalisation de l'apprentissage d'un CLOM, notre grille se base sur une méthode de calcul qui tient compte à la fois :

- de la présence/absence de chaque propriété d'adaptation de composantes ou ressources-4A du scénario du CLOM ;

- de la valeur de pondération attribuée à chaque propriété d'adaptation de composantes ou ressources-4A dans le scénario du CLOM, dont les règles sont présentées à la section suivante.

En raison du grand nombre de propriétés composant notre grille, nous allons présenter uniquement les propriétés liées à un exemple de composante adaptable de haut niveau (liste des activités d'apprentissage), un exemple de composante liée aux activités d'apprentissage (moment de démarrage de l'activité d'apprentissage) et aux ressources-4A de type outil$4 \mathrm{~A}$ - l'ensemble de la grille étant disponible dans https://drive.google.com/file/d/0Bw9IV9UOtZd7WlBWVjA5MmY1RWM/ view?usp=sharing. Comme le montre l'extrait de notre grille présenté aux 
Rim BEJAOUI, Gilbert PAQUETTE, Josianne BASQUE, France HENRI

tableaux 1, 2 et 3, un utilisateur de la grille détermine d'abord la présence ou l'absence des propriétés de personnalisation de l'apprentissage en remplissant la colonne intitulée "Oui/Non». Dans cette dernière, il attribue à chaque propriété la valeur «1", signifiant que la propriété est présente dans le CLOM, ou la valeur « 0 », signifiant que la propriété est absente du CLOM. Une fois cette colonne remplie, un score de personnalisation de l'apprentissage (PA) pourra être calculé (voir section 3.3).

Par exemple, dans le tableau 1, on retrouve cinq possibilités pour l'adaptation de la liste des activités d'apprentissage, selon que celle-ci est fixe, ou encore restreinte ou étendue par un agent externe ou par l'apprenant. Il en est de même dans le tableau 2 quant à la durée d'une activité donnée. 
Sticef - Numéro Spécial - Recherches actuelles sur les MOOC Recueil 2017

Tableau 1 - Aperçu de la grille d'analyse (1/3) : Propriétés d'adaptation de la liste des activités d'apprentissage du scénario pédagogique de CLOMp

\begin{tabular}{|c|l|l|l|}
\hline P & \multicolumn{2}{|c|}{ Propriété } & \multicolumn{1}{c|}{ Définition } \\
0 & $\begin{array}{l}\text { Liste des activités d'ap- } \\
\text { prentissage fixe }\end{array}$ & $\begin{array}{l}\text { La liste des activités d'apprentis- } \\
\text { sage n'est pas modifiable en cours } \\
\text { de diffusion. }\end{array}$ & \\
\hline 1 & $\begin{array}{l}\text { Sous-liste des activités } \\
\text { d'apprentissage assignée } \\
\text { par un agent externe }\end{array}$ & $\begin{array}{l}\text { Un agent externe peut assigner à } \\
\text { l'apprenant, en cours de diffusion, } \\
\text { une sous-liste des activités d'ap- } \\
\text { prentissage à réaliser. }\end{array}$ & \\
\hline 2 & $\begin{array}{l}\text { Sous-liste des activités } \\
\text { d'apprentissage au choix } \\
\text { de l'apprenant }\end{array}$ & $\begin{array}{l}\text { L'apprenant peut choisir, en } \\
\text { cours de diffusion, les activités } \\
\text { d'apprentissage à réaliser dans la } \\
\text { liste. }\end{array}$ & \\
\hline 3 & $\begin{array}{l}\text { Liste des activités d'ap- } \\
\text { prentissage extensible } \\
\text { par un agent externe }\end{array}$ & $\begin{array}{l}\text { Un agent externe peut ajouter, en } \\
\text { cours de diffusion, de nouvelles } \\
\text { activités d'apprentissage à la liste. }\end{array}$ & \\
\hline 4 & $\begin{array}{l}\text { Liste des activités d'ap- } \\
\text { prentissage extensible } \\
\text { par l'apprenant }\end{array}$ & $\begin{array}{l}\text { L'apprenant peut ajouter, en } \\
\text { cours de diffusion, de nouvelles } \\
\text { activités d'apprentissage à la liste. }\end{array}$ & \\
\hline
\end{tabular}

Tableau 2 - Aperçu de la grille d'analyse (2/3) : propriétés d'adaptation du moment de démarrage de l'activité d'apprentissage dans un scénario pédagogique de CLOMp

\begin{tabular}{|c|l|l|c|}
\hline \multicolumn{2}{|c|}{ Propriété } \\
0 & $\begin{array}{l}\text { Moment de démar- } \\
\text { rage fixe }\end{array}$ & $\begin{array}{l}\text { Définition } \\
\text { untivité d'apprentissage débute à } \\
\text { modification en cours de diffusion. }\end{array}$ & Oui/No \\
\hline 1 & $\begin{array}{l}\text { Moment de démar- } \\
\text { rage assigné par un } \\
\text { agent externe avec } \\
\text { date limite }\end{array}$ & $\begin{array}{l}\text { Un agent externe peut assigner, en } \\
\text { cours de diffusion, un moment de } \\
\text { démarrage à l'activité d'apprentis- } \\
\text { sage, en respectant une date limite } \\
\text { de démarrage à ne pas dépasser. }\end{array}$ & \\
\hline 2 & $\begin{array}{l}\text { Moment de démar- } \\
\text { rage assigné par un } \\
\text { agent externe sans } \\
\text { date limite }\end{array}$ & $\begin{array}{l}\text { Un agent externe peut assigner, en } \\
\text { cours de diffusion, un moment de } \\
\text { démarrage à l'activité d'apprentis- } \\
\text { sage sans date limite de démarrage. }\end{array}$ & \\
\hline
\end{tabular}


Rim BEJAOUI, Gilbert PAQUETTE, Josianne BASQUE, France HENRI

\begin{tabular}{|c|l|l|c|}
\hline \multicolumn{2}{|c|}{ Propriété } & \multicolumn{2}{c|}{ Définition } \\
P & $\begin{array}{l}\text { Moment de démar- } \\
\text { rage au choix de l'ap- } \\
\text { prenant avec date } \\
\text { limite }\end{array}$ & $\begin{array}{l}\text { L'apprenant peut choisir, en cours } \\
\text { de diffusion, un moment de démar- } \\
\text { rage pour l'activité d'apprentissage } \\
\text { en respectant une date limite de } \\
\text { démarrage à ne pas dépasser. }\end{array}$ & \\
\hline 4 & $\begin{array}{l}\text { Moment de démar- } \\
\text { rage au choix de l'ap- } \\
\text { prenant sans date } \\
\text { limite }\end{array}$ & $\begin{array}{l}\text { L'apprenant peut choisir, en cours } \\
\text { de diffusion, un moment de démar- } \\
\text { rage pour l'activité d'apprentissage } \\
\text { sans date limite de démarrage. }\end{array}$ & \\
\hline
\end{tabular}

Par contre, dans le tableau 3, pour les outils-4A, on se contente d'observer la présence ou l'absence des différents types d'outil d'assistance et d'aide à l'autogestion de l'apprentissage.

Tableau 3 - Aperçu de la grille d'analyse (3/3) : 4 exemples d'outils-4A du scénario pédagogique de CLOMp

\begin{tabular}{|c|l|l|l|}
$\mathbf{P}$ & \multicolumn{1}{|c|}{ Outil-4A } & \multicolumn{1}{|c|}{ Définition } & \multicolumn{1}{|c|}{$\begin{array}{c}\text { Oui/No } \\
\text { n }\end{array}$} \\
\hline 4 & $\begin{array}{l}\text { Agrégation de } \\
\text { ressources péda- } \\
\text { gogiques et diffu- } \\
\text { sion }\end{array}$ & $\begin{array}{l}\text { Outil permettant la collecte, } \\
\text { l'agrégation et la redistribution de } \\
\text { ressources pédagogiques (p. ex. } \\
\text { feedreader, gRSShopper). }\end{array}$ & \\
\hline 4 & $\begin{array}{l}\text { Autodiagnostic } \\
\text { des compétences }\end{array}$ & $\begin{array}{l}\text { Outil aidant l'apprenant à évaluer } \\
\text { ses forces et ses faiblesses en re- } \\
\text { gard des compétences visées par } \\
\text { le CLOM (p. ex. Compétences+). }\end{array}$ & \\
\hline 4 & $\begin{array}{l}\text { Co-création et } \\
\text { travail collabora- } \\
\text { tif }\end{array}$ & $\begin{array}{l}\text { Outil permettant de créer et de } \\
\text { modifier des documents en ligne } \\
\text { et de travailler en équipe, en } \\
\text { temps réel ou en différé (p. ex. } \\
\text { Wikispaces, GoogleDocs). }\end{array}$ & \\
\hline 4 & $\begin{array}{l}\text { Évaluation des } \\
\text { apprentissages } \\
\text { pair à pair }\end{array}$ & $\begin{array}{l}\text { Outil permettant l'évaluation des } \\
\text { productions de l'apprenant par } \\
\text { ses pairs. }\end{array}$ & \\
\hline
\end{tabular}

\subsection{Pondération des propriétés techno- pédagogiques de la grille d'analyse}

Dans notre ontologie et dans notre grille d'analyse, un niveau d'impact (valeur de pondération) sur la personnalisation de l'apprentissage est associé à chaque propriété d'adaptation de composante ou à chaque exemple (instance) de ressource-4A. Les critères de pondération que nous avons définis pour déterminer ces différents 
niveaux d'impact vont d'une propriété n'ayant aucun impact (niveau 0) à une propriété ayant un impact très important (niveau 4), en passant par des propriétés ayant peu d'impact (niveau 1), moyennement d'impact (niveau 2) et assez d'impact (niveau 3).

Il est à noter que, dans notre échelle, les propriétés d'adaptation des composantes du CLOM ont des niveaux d'impact pouvant aller de « 0 » à « 4 », alors que les ressources- $4 A$ ont des niveaux d'impact pouvant aller de « 2 » à « 4 ». Les niveaux d'impact que nous avons établis pour les propriétés d'adaptation de composantes et pour les ressources-4A sont justifiés dans les sous-sections suivantes.

\subsubsection{Variables de pondération des propriétés d'adaptation des composantes du CLOMp}

Les valeurs de pondération des propriétés d'adaptation des composantes $d u$ CLOMp sont déterminées en tenant compte des variables suivantes:

- l'acteur qui effectue l'action d'adaptation : lorsque l'action d'adaptation est prise en charge par l'apprenant ou un groupe d'apprenants, la valeur de pondération est plus élevée que si elle est sous la responsabilité d'un agent externe (facilitateur ou agent logiciel); par exemple, l'adaptation de la durée de l'activité d'apprentissage par l'apprenant est d'un degré de personnalisation plus élevé que son adaptation par un facilitateur. Bien que la personnalisation par un agent externe puisse être importante, nous postulons que celle effectuée par l'apprenant l'est davantage, car plus proche de ses besoins tout en favorisant le développement de son autonomie ;

- les actions d'adaptation : certaines sont considérées d'un degré de personnalisation plus élevé que d'autres parce qu'elles impliquent un acte de conception ou de production par le responsable de la personnalisation comparativement à un acte de simple choix parmi des objets prédéfinis dans le CLOMp; par exemple, pour les composantes adaptables Liste des compétences, Liste des activités d'apprentissage, Listes des ressources à utiliser et Liste des ressources à produire, l'action d'ajouter des éléments à une liste prédéfinie présente un degré de personnalisation plus élevé que celle de choisir une sous-liste à partir de cette liste ;

- les objets sur lesquels l'action d'adaptation est réalisée : certains objets ont une valeur contributoire plus élevée que d'autres dans l'acte d'apprentissage. Par exemple, pour la composante adaptable Plateforme de CLOM, l'action de permettre de choisir les fonctionnalités offertes aux apprenants présente une action de personnalisation plus 
élevée que celle de choisir le format d'affichage (skin) (couleurs, formes, texture);

- l'existence d'une contrainte de temps, de taille ou d'ampleur de modification applicable à une composante adaptable : l'adaptation des composantes adaptables Ordonnancement des activités, Durée, Moment de démarrage et Mode de constitution d'un groupe d'apprenants peut être sujette à de telles contraintes. Si l'action d'adaptation permise n'impose pas de contrainte d'ampleur de modification, de temps ou de taille, elle est considérée d'un degré de personnalisation plus élevé que la même action d'adaptation accomplie avec une contrainte d'ampleur de modification, de temps ou de taille. Par exemple, la constitution d'un groupe d'apprenants sans limite de taille est d'un degré de personnalisation plus élevé que la constitution d'un groupe d'apprenants avec limite de taille.

\subsubsection{Fonctions de pondération des ressources-4A}

Les valeurs de pondération des ressources-4A du CLOM sont fondées sur notre adaptation de la typologie des fonctions supportées par les objets médiatisés définie par Peraya (Peraya, 2008). Sur la base de plusieurs travaux, ce dernier propose huit fonctions génériques d'un dispositif de formation et de communication qui sont présentées dans la deuxième colonne du tableau 4. Nous avons adapté ces huit fonctions génériques en renommant certaines d'entre elles (voir colonne "Fonctions d'une ressource- $4 \mathrm{~A} »)$ et en scindant en deux catégories distinctes la quatrième fonction générique "Gestion et planification", à savoir Gestion $(G)$ (fonction de gestion pouvant être offerte par une ressource-4A) et Planification (Pl) (fonction de planification pouvant être offerte par une ressource-4A). La raison pour laquelle nous avons scindé ces deux fonctions est qu'elles sont indépendantes l'une de l'autre: une ressource$4 A$ (par ex. un outil de marquage social et d'annotation de ressources pédagogiques comme Diigo ou Delicious) peut fournir une fonction de Gestion $(G)$, sans pour autant fournir une fonction de Planification (Pl), et vice-versa. 
Sticef - Numéro Spécial - Recherches actuelles sur les MOOC Recueil 2017

Tableau 4 - Fonctions supportées par les ressources-4A du scénario pédagogique de CLOMp (adapté de Peraya, 2008)

\begin{tabular}{|c|c|}
\hline $\begin{array}{l}\text { Fonctions } \\
\text { d'une res- } \\
\text { sources-4A }\end{array}$ & $\begin{array}{l}\text { Fonctions supportées par les objets médiatisés } \\
\text { (Peraya, 2008) }\end{array}$ \\
\hline $\begin{array}{l}\text { Consultation } \\
\text { d'information } \\
\text { (C) }\end{array}$ & $\begin{array}{l}\text { 1. Information (donner aux apprenants des ressources } \\
\text { pédagogiques, donc des connaissances déjà constituées). }\end{array}$ \\
\hline $\begin{array}{l}\text { Socialisation } \\
\text { (S) }\end{array}$ & 2. Interaction sociale (communiquer, collaborer). \\
\hline Production (Pr) & $\begin{array}{l}\text { 3. Production (transformer des ressources en connais- } \\
\text { sances au cours d'un processus matériel, symbolique et } \\
\text { cognitif instrumenté). }\end{array}$ \\
\hline Gestion (G) & \multirow{2}{*}{$\begin{array}{l}\text { 4. Gestion (des apprenants, des groupes, des dossiers sco- } \\
\text { laires, etc.) et planification (des acteurs, des ressources, } \\
\text { des activités d'apprentissage). }\end{array}$} \\
\hline $\begin{array}{l}\text { Planification } \\
\text { (P1) }\end{array}$ & \\
\hline Tutorat (T) & $\begin{array}{l}\text { 5. Soutien et accompagnement (pratique tutorale rela- } \\
\text { tive aux domaines technique, cognitif, organisationnel } \\
\text { et méthodologique, socio-affectif et rationnel). }\end{array}$ \\
\hline $\begin{array}{l}\text { Métareflexivité } \\
\text { (M) }\end{array}$ & $\begin{array}{l}\text { 6. Émergence et systématisation de l'activité d'apprentis- } \\
\text { sage métaréflexive (considérée comme une aide à } \\
\text { l'apprentissage). }\end{array}$ \\
\hline Évaluation (E) & 7. Auto- et hétéro-évaluation. \\
\hline Awareness (A) & $\begin{array}{l}\text { 8. A wareness (gérer et faire circuler les signes de la pré- } \\
\text { sence à distance de chaque intervenant dans } \\
\text { l'environnement). }\end{array}$ \\
\hline
\end{tabular}

L'adaptation de la typologie de Peraya a servi à classifier les différentes fonctions des ressources- $4 A$ visant à l'assister dans sa démarche d'apprentissage autogérée, comme suit :

- la fonction de consultation d'information (C) a moyennement d'impact sur la personnalisation de l'apprentissage (PA) dans le CLOMp ;

- les fonctions de socialisation (S), de gestion (G) et de tutorat (T) ont assez d'impact sur la personnalisation de l'apprentissage (PA) dans le CLOMp ;

- les fonctions de production (Pr), de métareflexivité (M), de planification ( $\mathrm{Pl})$, d'évaluation (E) et d'awareness (A) ont un impact très important sur la personnalisation de l'apprentissage (PA) dans le CLOMp. 
Ainsi, nous postulons que toutes les fonctions permettant le développement de l'autonomie de l'apprenant ( $\mathrm{Pr}, \mathrm{M}$, Pl et $\mathrm{A}$ ) ont un impact plus important que celles permettant de lui fournir de l'assistance $(\mathrm{S}, \mathrm{G}$ et $\mathrm{T})$, ces dernières ayant un impact plus important que la simple fonction de consultation d'information (C).

La valeur de pondération d'une ressource-4A correspond à la valeur maximale dans l'ensemble des pondérations associées aux fonctions offertes par cette ressource. Par exemple, si une ressource-4A offre les fonctions de l'ensemble $\{\mathrm{C}, \mathrm{Pr}, \mathrm{G}, \mathrm{Pl}, \mathrm{M}\}$, alors sa valeur de pondération est $\operatorname{Max}\{2,4,3,4,4\}=4$. Nous obtenons ainsi les valeurs de pondération associées aux ressources-4A présentées dans l'extrait de la grille d'analyse au tableau 3 présenté plus haut.

\subsection{Calcul du score de personnalisation de I'apprentissage d'un CLOM}

Une fois que l'évaluateur aura attribué une valeur « 0 » ou « 1 » à chaque propriété de personnalisation de l'apprentissage de notre grille d'analyse, un score de personnalisation de l'apprentissage (PA) peut être calculé grâce la formule suivante :

\section{Score $P A=$}

Score AdapCHN+ Score AdapCAA + Score Ressources-4A

où :

- Score PA correspond au score de personnalisation de l'apprentissage (PA) du CLOM ;

- Score AdapCHN correspond au score d'adaptabilité des composantes de haut niveau (CHN) du CLOM, c'est-à-dire au niveau du scénario pédagogique global ;

- Score AdapCAA correspond au score total d'adaptabilité des composantes liées à chacune des activités d'apprentissage (CAA) du CLOM ;

- Score Ressources-4A correspond au score de présence de ressources d'assistance et d'aide à l'autogestion de l'apprentissage (Ressources-4A) dans le CLOM.

Ces trois scores sont calculés en tenant compte des critères de pondération présentés à la section 3.2 
La valeur de Score AdapCHN correspond à la somme des valeurs de pondération de toutes les propriétés d'adaptation des composantes de haut niveau $(\mathrm{CHN})$ présentes dans le CLOMp (c'est-à-dire ayant la valeur «1» dans la grille d'analyse) divisée par la valeur maximale de Score AdapCHN (présentée plus bas dans le tableau 5). Or, les propriétés d'adaptation des CHN d'un CLOM présentes dans notre grille incluent des propriétés d'adaptation de CHN par un agent externe (AE) (par ex. Liste des activités d'apprentissage extensible par un agent externe) et des propriétés d'adaptation de CHN par l'apprenant (par ex. Ordonnancement des activités d'apprentissage modifiable totalement par l'apprenant). Ce qui permet de calculer un score d'adaptabilité des $\mathrm{CHN}$ par un agent externe (Score AdapCHN_AE) et un score d'adaptabilité des CHN par l'apprenant (Score AdapCHN_AP) d'une façon analogue au calcul de Score AdapCHN.

Le calcul du score d'adaptabilité des composantes liées aux activités d'apprentissage (CAA) se fait en calculant les scores pour chacune des activités et en les combinant pour l'ensemble des activités du scénario.

La valeur de Score Ressources- $4 A$ correspond à la somme des quatre sous-scores Score Guides-4A, Score Instruments-4A, Score Outils-4A et Score Services- $4 A$ tenant compte des pondérations associées aux éléments ayant la valeur «1» dans les quatre listes de ressources-4A correspondantes.

\subsection{Règles d'exclusion mutuelle des propriétés d'adaptation des composantes du CLOM}

La sélection effectuée par l'évaluateur d'un CLOM de la valeur de présence/absence des propriétés d'adaptation des CHN et des CAA du CLOM doit se faire conformément à 60 règles définissant les relations d'exclusion mutuelle qui existent entre les propriétés d'une même composante (voir

à https://drive.google.com/file/d/0Bw9IV9UOtZd7WlBWVjA5MmY1RWM/ view?usp=sharing). Par exemple, si pour la composante adaptable Liste des compétences, l'évaluateur attribue la valeur «1" à la propriété Liste des compétences fixe, alors la valeur de chacune des autres propriétés pour cette composante doit être égale à « 0 ». Si, par contre, l'évaluateur attribue la valeur « 0 » à l'instance Liste des compétences fixe, il devra alors attribuer la valeur «1» à, au moins, l'une des autres instances de la classe Liste des compétences. 
En cours d'analyse, il n'existe pas de règles associées à la sélection, par l'évaluateur, des valeurs de présence/absence des ressources-4A dans le CLOM analysé, puisque tous les exemples de ressources-4A (guides-4A, instruments- $4 \mathrm{~A}$, outils- $4 \mathrm{~A}$ et services- $4 \mathrm{~A}$ ) de notre grille peuvent être présents, en même temps, dans le CLOM ou absents de ce dernier.

\subsection{Score de personnalisation de l'apprentissage maximal d'un CLOM}

Le tableau 5 présente les valeurs maximales des scores de personnalisation de l'apprentissage d'un CLOM incluant $N$ activités d'apprentissage. Ces scores maximaux permettent d'interpréter les divers scores de personnalisation obtenus lorsqu'un CLOM est analysé à l'aide de notre grille.

Tableau 5 - Scores maximaux de personnalisation de l'apprentissage dans un CLOM ayant $N$ activités d'apprentissage

\begin{tabular}{|l|c|}
\hline \multicolumn{1}{|c|}{$\begin{array}{c}\text { Personnalisation de l'apprentissage } \\
\text { dans un CLOM } \\
\text { Personnalisation de l'apprentissage } \\
\text { (PA) globale }\end{array}$} & $\mathbf{3 6}+\left(\mathbf{1 2 8}{ }^{*} \mathbf{N}\right)+\mathbf{9 0}$ \\
\hline 1. Adaptabilité des CHN & $\mathbf{3 6}$ \\
\hline 1.1 Adaptabilité des CHN par un agent externe & 14 \\
\hline 1.2 Adaptabilité des CHN par l'apprenant & 22 \\
\hline 2. Adaptabilité des CAA & $\mathbf{1 2 8}{ }^{*} \mathbf{N}$ \\
\hline 2.1 Adaptabilité des CAA par un agent externe & $49{ }^{*} \mathrm{~N}$ \\
\hline 2.2 Adaptabilité des CAA par l'apprenant & $72{ }^{*} \mathrm{~N}$ \\
\hline 3. Ressources-4A & $\mathbf{9 0}$ \\
\hline 3.1 Guides-4A & 6 \\
\hline 3.2 Instruments-4A & 8 \\
\hline 3.3 Outils-4A & 64 \\
\hline 3.4 Services-4A & 12 \\
\hline
\end{tabular}


Sticef - Numéro Spécial - Recherches actuelles sur les MOOC Recueil 2017

\subsection{Interprétation des scores de personnalisation de I'apprentissage du CLOM}

Les cinq conditions présentées dans le tableau 6, utilisant les scores maximaux présentés au tableau 5, permettent d'interpréter chacun des scores de personnalisation de l'apprentissage d'un CLOM présentés dans la section 3.5 .

Dans le tableau 6, « $\mathrm{X}$ » peut représenter l'une ou l'autre des valeurs suivantes: "Personnalisation de l'apprentissage», "Adaptabilité des CHN », «Adaptabilité des CHN par un agent externe », "Adaptabilité des CHN par l'apprenant", "Adaptabilité des CAA», "Adaptabilité des CAA par un agent externe», "Adaptabilité des CAA par l'apprenant», «Ressources-4A», «Guides-4A », «Instruments-4A », «Outils-4A » et «Services- $4 \mathrm{~A} »$.

Tableau 6 - Interprétation des scores de personnalisation de l'apprentissage d'un CLOM

\begin{tabular}{|l|l|}
\multicolumn{1}{|c|}{$\begin{array}{c}\text { Si ScoreMax }(\mathbf{x}) / \\
\text { Score }(\mathbf{x}) \boldsymbol{\epsilon}\end{array}$} & \multicolumn{1}{c|}{ Alors x est ... } \\
\hline$[0,0.125[$ & absent du CLOM \\
\hline$[0.125,0.375[$ & peu présent dans le CLOM \\
\hline$[0.375,0.625]$ & $\begin{array}{l}\text { moyennement présent dans le } \\
\text { CLOM }\end{array}$ \\
\hline$[0.625,0.875[$ & assez présent dans le CLOM \\
\hline$[0.875,1]$ & très présent dans le CLOM \\
\hline
\end{tabular}

\section{Résultats d'application de la grille d'analyse sur le CLOM DS106}

Cette section montre les résultats d'application de notre grille d'analyse du degré de personnalisation dans le CLOM intitulé Digital Storytelling (DS106). Nous avons choisi cet exemple en raison de sa simplicité, car il ne propose qu'une seule activité d'apprentissage. Nous le présentons dans la section 4.1. Nous présentons, par la suite, les résultats de son analyse à l'aide de notre grille à la section 4.2.

\subsection{Présentation du CLOM DS106}

Le CLOM DS106, dont l'organisme incubateur est l'Université Mary Washington située dans l'État de Virginie aux États-Unis, a été créé par le professeur Jim Groom et diffusé pour la première fois à l'automne 2010. Il a pour objectif le développement de compétences dans l'utilisation 
d'outils de mise en réseau, de partage, de narration et d'expression créative. Depuis son lancement, le cours a subi plusieurs itérations et est diffusé plusieurs fois par année. Actuellement, il existe une version du cours en diffusion continue (sans date de début ni de fin), soutenue par un site web (http://ds106.us/open-course), dans lequel une base de données contenant les productions des participants, nommée Assignement Bank, constitue l'élément central. L'activité d'apprentissage principale de DS106 est l'élaboration de productions d'expression créative libres, à l'aide de divers outils proposés aux participants dans le site web du cours ou d'outils qu'ils peuvent choisir (par ex. logiciels de retouche, de traitement et de dessin; outils de production audio/vidéo ; plateformes de blogging, de réseautage social ou d'hébergement de contenu multimédia). À l'aide d'un formulaire disponible sur le site web DS106, les apprenants sont encouragés, en publiant une production, à décrire leur processus d'élaboration, permettant ainsi aux autres apprenants de le reproduire ou de le modifier. Le site web dispose aussi d'une variété d'outils, entre autres, une station de radio qui diffuse en continu les productions des participants et débats autour de ces dernières, ainsi qu'un outil de mixage permettant de combiner entre elles des productions disponibles dans la banque du cours. Dans ce CLOM, il est postulé que l'évaluation de l'apprentissage est fondée sur les commentaires fournis par les pairs. Les participants sont ainsi incités à partager leurs productions et à élaborer des commentaires sur les productions des autres membres de la communauté. Les activités de publication et de socialisation se font sur le site web DS106 ainsi que sur de nombreuses autres plateformes de réseautage social et d'hébergement de contenu multimédia, dont Twitter, Googlet, Youtube et SoundCloud.

\subsection{Résultats d'analyse du CLOM DS106}

Le tableau 7 montre les résultats d'analyse du degré de personnalisation de l'apprentissage de DS106.

Tableau 7 - Résultats d'analyse du degré de personnalisation de l'apprentissage de DS106

\begin{tabular}{|l|l|c|}
\hline $\begin{array}{l}\text { Personnalisation de l'apprentissage } \\
\text { dans DS106 }\end{array}$ & \multicolumn{1}{|c|}{$\begin{array}{c}\text { Score } \\
\text { Interpréta- } \\
\text { tion }\end{array}$} \\
\hline $\begin{array}{l}\text { Personnalisation de l'apprentissage } \\
\text { (PA) olnhale }\end{array}$ & $\begin{array}{l}42.9 \\
\%\end{array}$ & $\begin{array}{c}\text { Moyenne- } \\
\text { ment nré- }\end{array}$ \\
\hline 1. Adaptabilité des CHN & $\begin{array}{l}44.4 \\
\%\end{array}$ & $\begin{array}{c}\text { Moyenne- } \\
\text { ment nré- }\end{array}$ \\
\hline
\end{tabular}


Sticef - Numéro Spécial - Recherches actuelles sur les MOOC Recueil 2017

\begin{tabular}{|c|c|c|}
\hline $\begin{array}{l}\text { Personnalisation de lapprentissage } \\
\text { dans DS106 }\end{array}$ & Score & $\begin{array}{l}\text { Interpréta- } \\
\text { tion }\end{array}$ \\
\hline $\begin{array}{l}\text { 1.1 Adaptabilité des CHN par un agent ex- } \\
\text { terne }\end{array}$ & $0.0 \%$ & Absente \\
\hline 1.2 Adaptabilité des CHN par l'apprenant & $72.7 \%$ & Assez présente \\
\hline 2. Adaptabilité des CAA & $\begin{array}{l}46.0 \\
\%\end{array}$ & $\begin{array}{l}\text { Moyenne- } \\
\text { ment nré- }\end{array}$ \\
\hline $\begin{array}{l}\text { 2.1 Adaptabilité des CAA par un agent ex- } \\
\text { terne }\end{array}$ & $0.0 \%$ & Absente \\
\hline 2.2 Adaptabilité des CAA par l'apprenant & $69.4 \%$ & Assez présente \\
\hline 3. Ressources-4A & $\begin{array}{l}37.7 \\
\%\end{array}$ & $\begin{array}{l}\text { Moyenne- } \\
\text { ment nré- }\end{array}$ \\
\hline 3.1 Guides-4A & $66.6 \%$ & Assez présents \\
\hline 3.2 Instruments- $4 \mathrm{~A}$ & $0.0 \%$ & Absents \\
\hline 3.3 Outils $-4 \mathrm{~A}$ & $37.5 \%$ & $\begin{array}{l}\text { Moyennement } \\
\text { présents }\end{array}$ \\
\hline 3.4 Services- $4 \mathrm{~A}$ & $50.0 \%$ & $\begin{array}{l}\text { Moyennement } \\
\text { présents }\end{array}$ \\
\hline
\end{tabular}

On observe dans ce tableau que la personnalisation de l'apprentissage est moyennement présente dans ce CLOM. En effet, l'adaptabilité des composantes de haut niveau (CHN) et l'adaptabilité des composantes liées à l'activité d'apprentissage (CAA) sont moyennement présentes dans DS106. Ce résultat s'explique du fait que l'adaptabilité par l'apprenant des $\mathrm{CHN}$ et des CAA y est assez présente. Alors que l'adaptabilité par un agent externe des CHN et des CAA en est absente. Les ressources-4A sont, quant à elles, moyennement présentes dans ce CLOM, avec des guides-4A assez présents, des instruments-4A absents, des outils-4A moyennement présents et des services-4A moyennement présents.

Si les concepteurs de DS106 désirent augmenter le niveau de personnalisation de l'apprentissage dans les futures sessions, ils pourraient assurer la présence des propriétés d'adaptabilité par un agent externe aussi bien celles de haut niveau (CHN) que celles liées à l'unique activité d'apprentissage (CAA) du cours, ainsi qu'augmenter la présence des ressources-4A manquantes, surtout en ce qui concerne les instruments- $4 \mathrm{~A}$, les outils-4A et les services-4A. 
Ces consignes spécifiques pourraient être émises par un environnement d'assistance à la conception de CLOM ayant pour fondement notre ontologie et intégrant notre grille d'analyse. C'est l'objectif du prototype d'assistance à la conception de CLOMp que nous avons développé.

\section{Résultats de la validation de la grille d'analyse}

Cette section présente d'abord le cadre et les résultats de la validation de la grille d'analyse réalisée par les quatre experts.

\subsection{Cadre de la validation de la grille d'analyse}

La méthode que nous avons mise en œuvre vise une «validation du concept». Elle a été menée avec deux professeurs-chercheurs et deux professionnels de recherche. Ces participants détenaient une expérience pratique de conception pédagogique et/ou une expertise de recherche en personnalisation de l'apprentissage, en ingénierie des ENA et/ou des CLOM, ou en pédagogie des ENA et/ou des CLOM. Ils ont été invités à répondre à un questionnaire qui leur a été transmis par courriel (voir à https://drive.google.com/open?id=0Bw9IV9UOtZd7dThoUnZKdVRnNlE) , accompagné de deux autres documents : un exemple d'application de la grille d'analyse et les règles d'exclusion mutuelle des propriétés d'adaptation des composantes du CLOM. Ils ont aussi reçu un glossaire définissant 74 termes utilisés dans la grille, accompagné d'un aperçu de notre ontologie (présentée à la section 2).

Le questionnaire invitait les experts à se prononcer sur les différents aspects de notre grille d'analyse, soit en expliquant leur position, soit en indiquant leur niveau d'accord par rapport :

- (1) à l'importance de la personnalisation de l'apprentissage dans un CLOM ;

- (2) aux propriétés, identifiées et définies dans notre grille comme pouvant soutenir la personnalisation de l'apprentissage dans un CLOM ;

- (3) au regroupement de ces propriétés, leur pertinence et leur niveau d'impact sur la personnalisation de l'apprentissage dans un CLOM, et

- (4) à la méthode de calcul et d'interprétation des scores de personnalisation de l'apprentissage d'un CLOM utilisée dans notre grille d'analyse.

\subsection{Synthèse des réponses des experts au questionnaire de validation de la grille}


Concernant la personnalisation de l'apprentissage, les experts ont estimé que cette dernière est d'une importance capitale dans les ENA en général, et en particulier dans les CLOM, notamment pour:

- capitaliser les acquis des recherches en personnalisation de l'apprentissage (des ENA) des 30 dernières années;

- aider à motiver les apprenants qui peuvent se sentir perdus dans la masse et peu engagés dans le processus d'apprentissage et donc lutter contre le décrochage;

- rendre l'apprentissage dans les CLOM plus efficace, notamment en ciblant plus spécifiquement les besoins/difficultés/objectifs propres à un apprenant et faire face au manque d'interaction avec le tuteur dans ces dispositifs.

Par ailleurs, deux des quatre participants à la validation ont jugé pertinente notre classification des propriétés identifiées et définies dans notre grille comme pouvant soutenir la personnalisation de l'apprentissage dans un CLOM. Un autre expert la trouve séduisante mais n'est pas convaincu de sa pertinence, car il trouve que la personnalisation n'y apparait pas assez centralement. Ce dernier déclare que cela dépend du modèle d'ingénierie sur la base duquel sont définies nos classes de propriétés. Par ailleurs, tous les experts se sont dits d'accord avec les variables accordant différents niveaux d'impact aux propriétés d'adaptation des composantes du CLOM et ont trouvé pertinente l'idée de s'appuyer sur les huit (8) fonctions génériques définies par Peraya et de leur faire correspondre les valeurs de pondération 2 à 4 des ressources-4A. En outre, les experts ont émis des propositions d'amélioration sur 4 des 21 propriétés d'adaptation des composantes de haut niveau du CLOM, notamment les propriétés d'adaptation de la plateforme de CLOM. Ils ont éprouvé des problèmes de compréhension sur 14 des 73 propriétés d'adaptation des composantes liées aux activités d'apprentissage du CLOM, notamment:

- le mot «sous-liste» utilisé dans toutes les propriétés qui concernent un objet de type liste dont un des experts ne comprenait pas la signification ;

- le terme réalisateur (de l'activité d'apprentissage) qu'un des experts a proposé de remplacer par acteur(s) ou participant(s);

- les expressions "type d'agent évaluateur", "type d'évaluation des apprentissages » et "type d'instrument d'évaluation des apprentissages » dans les propriétés d'adaptation du mode d'évaluation des apprentissages dont un des experts ne comprenait pas la signification ; 
- les propriétés d'adaptation de la liste des ressources à utiliser et les propriétés d'adaptation de la liste des ressources- $4 \mathrm{~A}$ au sujet desquelles un des experts a déclaré ne pas avoir compris la différence.

Par ailleurs, les experts ont proposé de modifier les fonctions/pondérations associées à 10 des 27 exemples de ressources-4A, notamment l'historique, la progression des activités d'apprentissage, l'exportation de données vers l'EPA, le plan de travail, le portfolio, le forum d'assistance, le profil de groupe, le profil personnel, le support technique pour l'utilisation de la plateforme de CLOM et l'animation de la plateforme de CLOM. Finalement, les quatre experts se sont dits d'accord avec la méthode de calcul et d'interprétation des scores de personnalisation de l'apprentissage d'un CLOM utilisée dans notre grille d'analyse.

Après avoir rempli le questionnaire sur les différents aspects de notre grille d'analyse, les experts ont été invités à nous communiquer leurs remarques et leur critique. Des échanges avec ces derniers ont donc eu lieu par écrit et par vidéoconférence. Nous avons jugé certains de leurs propos pertinents. D'autres l'étaient moins, car à notre avis ils dénotaient une compréhension incomplète de la grille en raison sans doute de la densité des informations présentées. Les principales critiques et améliorations à apporter à l'ontologie et à la grille d'analyse sont d'ordre terminologique ou sont liées aux fonctions/pondérations des ressources-4A. Elles incluent la révision de certains termes utilisés afin d'en améliorer la compréhension par l'évaluateur du CLOM, notamment l'appellation "composantes (adaptables) de haut niveau»; le raffinement des propriétés liées à la plateforme de CLOM quant à l'ampleur des choix offerts pour l'affichage du contenu (par ex. modifier le style du texte ou les thèmes) ou quant aux possibilités d'intégration d'outils externes dans la plateforme; le raffinement de la pondération des propriétés d'adaptation des sous-composantes du mode d'évaluation des apprentissages et du mode d'assistance et d'aide à l'autogestion de l'apprentissage et, finalement, la révision des fonctions/pondérations associées à certaines ressources-4A de notre grille. Dans la version de la grille présentée dans cet article, nous avons, comme proposé par les experts, modifié l'affectation des fonctions de certaines ressources-4A. Ces modifications n'ont pas eu d'effet sur le niveau d'impact sur la personnalisation de l'apprentissage associé à certaines ressources- $4 \mathrm{~A}$, alors qu'elles ont modifié le niveau d'impact d'autres ressources-4A comme 
suit: tous les exemples d'instruments-A de notre grille sont passés d'un niveau impact très important à un niveau d'impact moyennement important; les outils-4A «historique» et «progression des activités d'apprentissage " sont passés d'un niveau d'impact assez important à un niveau d'impact très important; l'outil-4A «forum d'assistance » est passé d'un niveau impact très important à un niveau d'impact assez important et le service-4A de «support technique pour l'utilisation de la plateforme de CLOM» est passé d'un niveau impact moyennement important à assez important.

\section{Conclusion}

Les travaux présentés dans cet article se veulent une contribution aux efforts menés pour combler le besoin de recherches sur la personnalisation de l'apprentissage dans les CLOM. Ainsi, cette contribution permet, d'une part, de clarifier les diverses propriétés de personnalisation de l'apprentissage qui peuvent s'appliquer dans ce type d'ENA et, d'autre part, de proposer une grille d'analyse de ces propriétés pouvant servir à guider le concepteur de CLOM dans sa prise de décision en matière de personnalisation de l'apprentissage. Nous pensons qu'il serait judicieux d'envisager l'intégration de notre grille d'analyse aux outils de développement de CLOM les plus répandus (par ex. l'outil Studio d'OpenEdx), afin d'évaluer son intérêt auprès de concepteurs pédagogiques en situation authentique et aussi d'évaluer si la présence des propriétés de personnalisation de l'apprentissage dans les CLOM a des effets positifs sur l'apprentissage et la persévérance des apprenants.

Nous projetons également d'étendre l'utilisation de la grille d'analyse pour évaluer le degré de personnalisation de l'apprentissage d'autres types d'ENA et d'étudier la possibilité d'adapter la grille pour en permettre l'utilisation par l'apprenant en vue d'analyser son propre environnement personnel d'apprentissage.

\section{Remerciements}

Ce projet a été mené dans le cadre de la préparation de la thèse de doctorat en informatique cognitive réalisée par Rim Bejaoui à l'Université TÉLUQ sous la direction des professeurs Gilbert Paquette et Josianne Basque, ainsi que dans le cadre du projet «Cours de masse en ligne et apprentissage personnalisé: le défi pédagogique des CLOM». Les auteurs désirent souligner l'aide financière à leurs travaux de la part du Conseil de recherche en sciences humaines du Canada (CRSH/SSHRC). 
Rim BEJAOUI, Gilbert PAQUETTE, Josianne BASQUE, France HENRI

\footnotetext{
${ }^{1}$ Dans l'ontologie, les classes sont représentées par un rectangle et les propriétés par un hexagone. Un lien de régulation $(\mathrm{R})$ peut relier tout type de classe ou individu à une propriété pour en définir le domaine, ou encore relier une propriété à tout type de classe ou individu ou type de donnée pour en définir le co-domaine. Un lien de spécialisation (S) relie une sous-classe à une classe plus générale ou relie une sous-propriété à une propriété plus générale. Un lien d'instanciation (I) relie un type de donnée à sa valeur (donnée).
}

\section{BIBLIOGRAPHIE}

(Alario-Hoyos et al., 2014)

Alario-Hoyos, C., Pérez-Sanagustín, M., Cormier, D. et Delgado-Kloos, C. (2014). Proposal for a conceptual framework for educators to describe and design MOOCs. Journal of Universal Computer Science, 20(1), 623.

(Baker et Surry, 2013)

Baker, F. W. et Surry, D. (2013). Open education designs: A taxonomy for differentiating and classifying open learning environments. Dans R. McBride et M. Searson (dir.), Proceedings of Society for Information Technology \& Teacher Education International Conference 2013 (p. 189-194). Chesapeake, VA: Association for the Advancement of Computing in Education (AACE). Repéré à http://www.editlib.org/p/48090/

(Bandura, 1977)

Bandura, A. (1977). Social learning theory. Englewood Cliffs, NJ : Prentice-Hall. (Version antérieure publiée en 1971. New York, NY : General Learning Press. Repéré à

http://www.jku.at/org/content/e54521/e54528/e54529/e178059/Bandura_SocialLea rningTheory_ger.pdf)

(Bandura, 1997)

Bandura, A. (1997). Self-efficacy: The exercise of control. New York, NY : Freeman.

(Blanco et al., 2013)

Blanco, Á. F., García-Peñalvo, F. J. et Sein-Echaluce, M. (2013). A methodology proposal for developing adaptive cMOOC. Dans F. J. Garcia-Peñalvo (dir.), Proceedings of the First International Conference on Technological Ecosystem for Enhancing Multiculturality (p. 553-558). New York, NY: ACM. Repéré à http://dl.acm.org/citation.cfm?id=2536621

(Bejaoui, 2017)

Bejaoui, Rim (2017). Assistance à la conception de cours en ligne ouverts et massifs soutenant un apprentissage personnalisé (thèse de doctorat en informatique cognitive, Télé-université, Québec, Canada). Repéré à http://rlibre.teluq.ca/1071/

(Clerc, 2014)

Clerc, F. (2014). Mise en place de la personnalisation dans le cadre des MOOCs (Rapport Master Recherche, Université Lyon 1, France). Repéré à http://elearningdev.univ-lyon1.fr/persua2mooc/manuals/rapport.pdf

(Fischer, 2007) 
Fischer, G. (2007). Meta-design: Expanding boundaries and redistributing control in design. Dans C. Baranauskas, P. Palanque, J. Abascal, S. D. J. Barbosa (dir.), Human-Computer Interaction - INTERACT 2007 (p. 193-206). Berlin, Heidelberg, Allemagne : Springer-Verlag. Repéré à http://link.springer.com/chapter/10.1007/9783-540-74796-3_19

(Fischer, 2012)

Fischer, G. (2012). Meta-design: Empowering all stakeholder as co-designers. Dans R. Luckin et. al. (dir.), Handbook of Design in Educational Technology (p. 135-145). New York, NY: Routledge. Repéré à http://13d.cs.colorado.edu/ gerhard/papers/2012/paper-handbook.pdf

(Fischer et Ostwald, 2002)

Fischer, G. et Ostwald, J. (2002). Seeding, evolutionary growth, and reseeding: Enriching participatory design with informed participation. Dans T. Binder, J. Gregory et I. Wagner (dir.), Proceedings of the Participatory Design Conference 2002 (p. 135-143). Repéré à http://13d.cs.colorado.edu/ Gerhard/papers/pdc2002-ser.pdf

(Halimi et al., 2014)

Halimi, K., Seridi-Bouchelaghem, H. et Faron-Zucker, C. (2014). An enhanced personal learning environment using social semantic web technologies. Interactive Learning Environments, 22(2), 165-187. doi: 10.1080/10494820.2013.788032

(Henning et al., 2014)

Henning, P. A., Heberle, F., Streicher, A., Zielinski, A., Swertz, C., Bock, J. et Zander, S. (2014). Personalized web learning: Merging open educational resources into adaptive courses for higher education. Dans M. Kravcik, O.C. Santos et J.G. Boticario (dir), Proceedings of the 4th International Workshop on Personalization Approaches in Learning Environments, PALE 2014 (p.55-62). CEUR Workshop Proceedings, vol. 1181. Repéré à http://ceur-ws.org/Vol1181/pale2014_proceedings.pdf\#page $=57$

(Henri, 2014)

Henri, F. (2014). Les environnements personnels d'apprentissage, étude d'une thématique de recherche en émergence. Revue STICEF, 21, 121-147. Repéré à http://sticef.univ-lemans.fr/num/vol2014/16-henri-

epa/sticef_2014_NS_henri_16.htm

(Hutchins, 1995, chap. 9)

Hutchins, E. (1995). Cognition in the Wild (chap. 9 : Cultural cognition, p. 353374). Cambridge, MA: MIT Press. Repéré à https://www.ida.liu.se/ nilda/CSTpapers/Hutchins.pdf

(Kravcik et al., 2014)

Kravcik, M., Santos, O. C. et Boticario, J. G. (2014). Preface. Dans M. Kravcik, O.C. Santos et J.G. Boticario (dir), Proceedings of the 4th International Workshop on Personalization Approaches in Learning Environments, PALE 2014 (p. 1-6). CEUR Workshop Proceedings, vol. 1181. Repéré à http://ceur-ws.org/Vol1181/pale2014_preface.pdf

(Miranda et al., 2013)

Miranda, S., Mangione, G. R., Orciuoli, F., Gaeta, M. et Loia, V. (2013). Automatic generation of assessment objects and remedial works for MOOCs. Dans 
Rim BEJAOUI, Gilbert PAQUETTE, Josianne BASQUE, France HENRI

International Conference on Information Technology Based Higher Education and Training, ITHET 2013 (p. 1-8). doi:10.1109/ITHET.2013.6671018

(Mott, 2010)

Mott, J. (2010). Envisioning the post-LMS era: The open learning network. Educause Quarterly, 33(1), 1-9.

(C. Paquette, 1992)

Paquette, C. (1992). Une pédagogie ouverte et interactive. Tome 1: L'approche. Montréal, Canada : Éditions Québec Amérique.

(Paquette, 2002a)

Paquette, G. (2002a). L'ingénierie pédagogique: pour construire l'apprentissage en réseau. Sainte-Foy, Canada: Presses de l'Université du Québec.

(Paquette, 2002b)

Paquette, G. (2002b). Modélisation des connaissances et des compétences: un langage graphique pour concevoir et apprendre. Sainte-Foy, Canada : Presses de l'Université du Québec. à http://books.google.ca/books?hl=fr\&lr=\&id=C4G4MVw4yrEC\&oi=fnd\&pg=PR15\& $\mathrm{dq}=$ paquette+2002+mod\%C3\%A9lisation+connaissances+comp\%C3\%A9tences\& ots=3Anwy9vZzx\&sig=phrKqoQqywDkoBBPEVhRnjhSCII

(Paquette et al., 2015)

Paquette, G., Mariño, O., Rogozan, D. et Léonard, M. (2015). Competency-based personalization for massive online learning. Smart Learning Environments, 2, 1-19. doi:10.1186/s40561-015-0013-z

(Paquette et al., 2007)

Paquette, G., Rosca, I., Mihaila, S. et Masmoudi, A. (2007). TELOS: A serviceoriented framework to support learning and knowledge management. Dans S. Pierre (dir.), E-Learning Networked Environments and Architectures (p. 79-109). Londres, U.K.: Springer. Repéré à http://link.springer.com/chapter/10.1007/978-184628-758-9_4

(Pea, 1993)

Pea, R. D. (1993). Practices of distributed intelligence and designs for education. Distributed cognitions: Psychological and educational considerations, 11, 47-87.

(Pedro et al., 2012)

Pedro, L., Santos, C., Almeida, S. et Koch-Grünberg, T. (2012). Building a shared Personal Learning Environment with SAPO Campus. Dans L. Pedro, C. Santos et S. Almeida (dir.), Personal Learning Environment (PLE) Conference Proceedings. Repéré à http://revistas.ua.pt/index.php/ple/article/view/1426/1312

(Peraya, 2008)

Peraya, D. (2008). Un regard critique sur les concepts de médiatisation et médiation: nouvelles pratiques, nouvelle modélisation. Les Enjeux de l'information et de la communication, Suppl. 2008. Repéré à https://archiveouverte.unige.ch/unige:17665

(Raposo-Rivas et al., 2015) 
Raposo-Rivas, M., Martinez-Figueira, E. et Sarmiento Campos, J.-A. (2015). A Study on the Pedagogical Components of MOOC. Comunicar Journal: Moocs in Education, 44. doi:10.3916/C44-2015-03

(Santos et al., 2014)

Santos, O. C., Boticario, J. G. et Pérez-Marín, D. (2014). Extending web-based educational systems with personalised support through User Centred Designed recommendations along the e-learning life cycle. Science of Computer Programming, 88, 92-109. doi:10.1016/j.scico.2013.12.004

(Shaw, 2013)

Shaw, C. J. (2013). System design and architecture of an online, adaptive, and personalized learning platform (Master of Science in Technology and Policy, Massachusetts Institute of Technology). Repéré à http://dspace.mit.edu/handle/1721.1/81128

(Siemens, 2004)

Siemens, G. (2004). Connectivism: A learning theory for the digital age. International Journal of Instructional Technology and Distance Learning, 2(1), 3-10.

(Szafir et Mutlu, 2013)

Szafir, D. et Mutlu, B. (2013). ARTFul: adaptive review technology for flipped learning. Dans Proceedings of the SIGCHI Conference on Human Factors in Computing Systems (p. 1001-1010). New York, NY: ACM. Repéré à http://dl.acm.org/citation.cfm?id=2466128

(USDE, 2010)

U.S. Department of Education (USDE). (2010). Transforming American Education: Learning Powered by Technology. National Educational Technology Plan 2010. Repéré à http://tech.ed.gov/wp-content/uploads/2013/10/netp2010.pdf

(Vygotski, 1978)

Vygotski, L. S. (1978). Mind in society: The development of higher mental process. Cambridge, MA : Harvard University Press.

(Yang et al., 2014)

Yang, D., Piergallini, M., Howley, I. et Rose, C. (2014). Forum thread recommendation for massive open online courses. Dans J. Stamper, Z. Pardos, M. Mavrikis, B. M. McLaren (dir.) Proceedings of 7th International Conference on Educational Data Mining (p. 257-260). Repéré à http://educationaldatamining.org/EDM2014/uploads/procs2014/short\%20papers/ 257_EDM-2014-Short.pdf

(Yousef et al., 2014)

Yousef, A. M. F., Chatti, M. A., Schroeder, U. et Wosnitza, M. (2014). What drives a successful MOOC? An empirical examination of criteria to assure design quality of MOOCs. Dans Proceedings of the 2014 IEEE 14th International Conference on Advanced Learning Technologies, ICALT (p. 44-48). IEEE Computer Society, Conference Pub. Services. Repéré à http://dl.acm.org/citation.cfm?id=2672199 